\title{
La terapia immunosoppressiva nel trapianto di rene
}

\section{Aris Tsalouchos' e Maurizio Salvadori²}

\begin{abstract}
Immunosuppressive therapy in renal transplantation

Immunosuppressive therapy in renal transplantation can be distinguished in induction therapy and maintenance therapy.

Induction therapy is an intense immunosuppressive therapy administered at the time of kidney transplantation to reduce the risk of acute allograft rejection. In general, the induction immunosuppressive strategies used at kidney transplant centers fall into one of these two categories. One strategy relies upon high doses of conventional immunosuppressive agents, while the other utilizes antibodies directed against T-cell antigens in combination with lower doses of conventional agents.

Maintenance immunosuppressive therapy is administered to almost all kidney transplant recipients to help prevent acute rejection and loss of the renal allograft. Although an adequate level of immunosuppression is required to dampen the immune response to the allograft, the level of chronic immunosuppression is decreased over time (as the risk of acute rejection decreases) to help lower the overall risk of infection and malignancy; these risks directly correlate with the degree of overall immunosuppression.

The optimal maintenance immunosuppressive therapy in kidney transplantation is not established. The major immunosuppressive agents that are available in various combination regimens are glucocorticoids (primarily oral prednisone), azathioprine, mycophenolate mofetil (MMF), enteric-coated mycophenolate sodium (EC-MPS), cyclosporine (in non-modified or modified [microemulsion] form), Tacrolimus, everolimus, rapamycin (sirolimus), and Belatacept.
\end{abstract}

\section{Keywords}

Induction therapy, Maintenance therapy, Mechanisms of action, Side effects, Calcineurin inhibitors

\section{Introduzione}

Dalle rassegne precedenti si evince che uno dei problemi principali in corso di trapianto di rene è quello di controllare la risposta immune. Allo scopo vengono impiegati farmaci immunosoppressori.

I farmaci immunosoppressori impiegati nel trapianto di rene possono essere distinti in due categorie secondo il momento in cui vengono impiegati. Si distinguono così una terapia di induzione e una terapia di mantenimento.

\section{Terapia di induzione}

La terapia di induzione è una terapia immunosoppressiva somministrata al momento del trapianto per ridurre i rischi di rigetto acuto. I farmaci impiegati più frequentemente si distinguono in due categorie o strategie di somministrazione.

Una strategia consiste nell'impiego ad alte dosi dei farmaci usati poi come terapia di mantenimento.
Alternativamente, e più frequentemente, sono usati anticorpi mono o policlonali rivolti verso gli antigeni delle cellule $\mathrm{T}$. Vengono anche usati anticorpi monoclonali diretti verso l'attivazione delle cellule $\mathrm{T}$.

Molti studi controllati, randomizzati, indicano che la terapia di induzione mediante anticorpi biologici è più efficace della terapia di induzione praticata mediante i soli farmaci convenzionali. ${ }^{1}$

'Aris Tsalouchos Azienda UsI Centro S.O.S. Nefrologia e Dialisi, Ospedale SS Cosma e Damiano. Via Cesare Battisti 2, 51017 Pescia, Italy

2Maurizio Salvadori, Professore di Nefrologia già Dirfettore Nefrologia e Trapianto. Azienda Ospedaliera Careggi, via Massicini I5, 500 I4 Fiesole Firenze, Italy

\section{Corrispondenza:}

Maurizio Salvadori, Professore di Nefrologia già Dirfettore Nefrologia e Trapianto. Azienda Ospedaliera Careggi, via Massicini I5, 50014 Fiesole Firenze, Italy

E-mail: maurizio.salvadoril@gmail.com 
Gli anticorpi impiegati nella terapia di induzione sono anticorpi anti-linfocitari o anticorpi anti-recettore dell' interleuchina 2 (IL-2R).

\section{Anticorpi anti-linfocitari}

Possono essere policlonali o monoclonali. Gli anticorpi policlonali prodotti dal coniglio sono i più frequentemente usati (rATG).

Gli anticorpi monoclonali includono anche l'Alentuzumab (Campath 1-H) che è un anticorpo umanizzato anti-CD52. ${ }^{2}$

Il Rituximab è un anticorpo monoclonale anti-CD20, che induce una deplezione delle cellule B. È usato nel post-trapianto per il trattamento del rigetto acuto, ma anche come terapia di induzione in pazienti $\mathrm{AB} 0$ incompatibili o con anticorpi preformati anti-HLA del donatore. ${ }^{3}$

\section{Anticorpi anti-IL-2R}

La proliferazione delle cellule $\mathrm{T}$ dopo trapianto è mediata dalla produzione di IL-2. Anticorpi monoclonali antiIL-2R sono impiegati nella terapia di induzione. ${ }^{4}$

Per quanto riguarda l'impiego della terapia di induzione, i pazienti sono stratificati nel gruppo ad alto rischio di rigetto acuto e in quello a basso rischio di rigetto acuto.

Pazienti ad alto rischio di rigetto acuto. Si tratta di pazienti con uno o più mismatches per gli antigeni HLA, con presenza di anticorpi anti-HLA superiore al $10 \%$, con incompatibilità del gruppo sanguigno o con presenza di anticorpi diretti contro antigeni del donatore.

Per la maggioranza di tali pazienti è opportuna la somministrazione di rATG alla dose di 1 o $2 \mathrm{mg} / \mathrm{Kg}$ intraoperatoriamente. La somministrazione molte volte è poi ripetuta per tre giorni, associata alla terapia di mantenimento.

Diversi studi hanno documentato la superiorità della somministrazione diATG vs. la mancata somministrazione. ${ }^{5}$ Altri studi hanno documentato la superiorità delle rATG vs gli anticorpi anti-IL-2R. ${ }^{6}$

L'induzione con rATG è fatta con dosi da 1 a $6 \mathrm{mg}$ per volta e per una durata da 1 a 10 giorni. Sembra però ottimale un'induzione con un totale di $6 \mathrm{mg} / \mathrm{Kg} .{ }^{7}$ Dosi più elevate e per una durata maggiore aumentano il rischio d'infezioni e di sviluppo di linfomi.

L'Alentuzumab (Campath 1-H) come terapia di induzione è raramente usato (nel $10 \%$ dei trapianti negli Stati Uniti). Studi atti a confrontare l'efficacia di Alentuzumab vs. rATG o IL-2R antagonisti non hanno permesso di concludere in favore di Alentuzumab.

Una revisione sistematica di 21 studi ha permesso di concludere che Rituximab è utile nel caso di AB0 incompatibilità, mentre tale efficacia non sembra confermata nel caso di HLA incompatibilità.

Pazienti a basso rischio di rigetto acuto. In questi pazienti l'induzione può essere fatta sia con rATG, sia con anticorpi inibitori di IL-2R.

Le KDIGO del 2009 raccomandano l'uso di anticorpi anti IL-2R come terapia di prima scelta in pazienti a basso rischio. ${ }^{8}$

Gli anticorpi anti-IL-2R (Basiliximab) sono somministrati alle dosi di $20 \mathrm{mg}$ intraoperatoriamente, più $20 \mathrm{mg}$ in quarta giornata dall'intervento.

Alcuni studi documentano la superiorità delle rATG, mentre altri non confermano questo dato in pazienti a basso rischio.

In ogni caso, Basiliximab dovrebbe essere preferito a rATG in pazienti con leucopenia $(2000 / \mathrm{uL})$ ed in pazienti ipotesi.

L'efficacia degli anticorpi anti-IL-2R è riportata in una revisione sistematica della Cochrane che analizza 71 studi randomizzati, controllati. ${ }^{9}$ Confrontati con placebo, gli inibitori di IL-2R riducono l'incidenza di rigetto acuto. Confrontati con rATG gli inibitori di IL-2R hanno una più elevata incidenza di rigetti acuti, ma minori effetti collaterali, infezioni da citomegalovirus e neoplasie.

\section{Terapia di mantenimento}

La terapia immunosoppressiva di mantenimento è somministrata in quasi tutti i pazienti trapiantati di rene per prevenire l'insorgenza di rigetti acuti e la conseguente perdita dell'organo. Un adeguato livello di tale terapia è richiesto per ridurre la risposta immune nei confronti del trapianto.

Col tempo, dopo il trapianto, il livello dell'immunosoppressione di mantenimento è ridotto anche per impedire l'insorgenza di patologia infettiva e di neoplasie.

I farmaci usati nella terapia immunosoppressiva di mantenimento sono gli steroidi, gli antimetaboliti (azatioprina, micofenolato mofetil [MMF] e micofenolato di sodio gastroprotetto [EC-MPS]), gli inibitori della calcineurina (ciclosporina [CyA] e Tacrolimus [TAC]), gli inibitori del complesso mammalian target of rapamycin (mTOR) (sirolimus, everolimus), e più recentemente il Belatacept.

\section{Meccanismo di azione dei farmaci immunosoppressori}

\section{Corticosteroidi}

I corticosteroidi modulano la risposta immune regolando l'espressione genica. In particolare, sono regolati i geni per IL-1, IL-2, interferone gamma (IFN- $\gamma$ ), tumor necrosis factor alpha (TNF- $\alpha)$ e IL-6. 


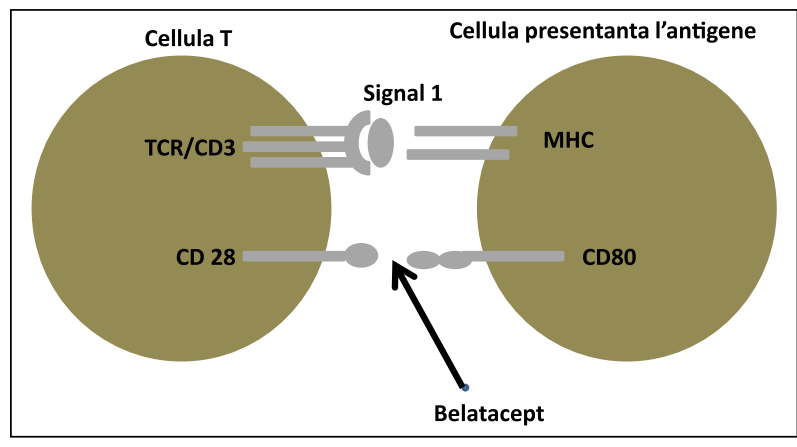

Figure I. Attivazione della cellula T attraverso due segnali.

\section{Azatioprina}

L'azatioprina inibisce la sintesi purinica e, di conseguenza, la sintesi di RNA. Impedisce la proliferazione linfocitaria dopo stimolazione antigenica. Inoltre, riduce il numero delle cellule monocitarie e granulocitarie e inibisce la proliferazione dei promielociti nel midollo.

\section{MMF ed EC-MPS}

In molti centri trapianto l'MMF, e successivamente la più efficace forma gastroprotetta (EC-MPS), hanno sostituito l'azatioprina. L'acido micofenolico è un inibitore selettivo della via di sintesi de novo delle purine. È un inibitore dell'inosina-fosfato deidrogenasi, e inibisce così in modo selettivo la proliferazione delle cellule B e T.

\section{Ciclosporina}

La ciclosporina, dopo essersi legata alla ciclofillina, inibisce la calcineurina. Questo porta al blocco dell'espressione genica delle citochine prodotta dalle cellule T. In particolare, si tratta di IL-2, IL-3, IL-4, IFN- $\gamma$ e TNF- $\alpha$. In particolare, la riduzione della concentrazione di IL-2 minimizza la risposta immune.

\section{Tacrolimus}

Pur avendo un meccanismo simile a quello della ciclosporina con inibizione della calcineurina, si tratta di una molecola strutturalmente assai differente. Per questo motivo non si lega alla ciclofillina, ma ad una proteina chiamata FK506-bindig protein (FKBP). Anche gli effetti collaterali del TAC sono simili, ma non uguali, a quelli indotti dalla ciclosporina.

\section{Inibitori di mTOR}

Sirolimus ed everolimus sono i principali farmaci appartenenti a questo gruppo. Si legano al complesso mTOR e prevengono la fosforilazione della p70S6 chinasi e la via di trasduzione mediata da IL-2. In aggiunta, gli inibitori della mTOR sono anche potenti inibitori del vascular endothelial growth factor (VEGF), il che spiega il loro ruolo nella prevenzione della crescita di molte forme di tumori.

Dato il potente effetto immunosoppressivo, gli mTOR inibitori sono spesso usati come agenti che permettono la riduzione o la completa eliminazione degli inibitori della calcineurina.

\section{Belatacept}

Il Belatacept è stato sviluppato per minimizzare il rischio cardiovascolare e la nefrotossicità associata agli inibitori della calcineurina.

La stimolazione immunitaria atta ad attivare la cellula $T$ necessita di un doppio segnale. Il primo segnale è dato dall'interazione degli antigeni del sistema maggiore d'istocompatibilità e il recettore della cellula T (TCR). Il secondo segnale è dato da molteplici stimoli. Il più importante ed efficace è dato dall'interazione fra il CD28 espresso sulla cellula T e il CD80 espresso sulla cellula presentante l'antigene (APC) (figura 1). A questo livello agisce il Belatacept come un antagonista competitivo, legandosi al CD80 e bloccando la stimolazione del CD28.

\section{Criteri di somministrazione della terapia di mantenimento}

La maggioranza dei centri trapianto usa una terapia di mantenimento con l'impiego simultaneo di tre farmaci.

- Un inibitore della calcineurina (ciclosporina o Tacrolimus)

- Un antimetabolita (azatioprina, MMF o EC-MPS)

- Gli steroidi

Questo è in accordo con le linee guida KDIGO. ${ }^{2}$

L'impiego degli mTOR inibitori come iniziale terapia di mantenimento non è condiviso da tutti nonostante $i$ buoni risultati raggiunti da alcuni centri. Molti centri preferiscono inserire gli mTOR inibitori in pazienti che sviluppano tossicità da inibitori della calcineurina.

Le stesse linee guida KDIGO suggeriscono la possibilità di discontinuare la somministrazione di steroidi per impedire l'insorgenza di effetti collaterali, almeno nei pazienti a basso rischio immunologico.

La terapia immunosoppressiva di mantenimento viene in genere iniziata al momento del trapianto o anche subito prima. EC-MPS è spesso somministrato prima del trapianto e poi continuato alla dose di $360 \mathrm{mg}$ due volte il giorno. Lo steroide è somministrato già durante l'intervento, spesso subito prima della reanastomosi alla dose di $500 \mathrm{mg}$ o1 g iv e poi continuato per os a dosi decrescenti fino a 5-10 
$\mathrm{mg}$ /die dopo un mese. L'inibitore della calcineurina (attualmente è preferito il TAC) viene iniziato il primo giorno dopo il trapianto alla dose di $0.08 \mathrm{mg} / \mathrm{Kg} /$ die nel caso del TAC per raggiungere un trough level di $7-10 \mathrm{mg} /$ $\mathrm{mL}$ nel primo mese e più basso successivamente.

In caso di oliguria, alcuni centri ritardano la somministrazione dell'inibitore della calcineurina fino a ottenere una significativa riduzione della creatinina.

\section{Scelta di un inibitore della calcineurina}

CyA e TAC hanno lo stesso meccanismo di azione, anche se col passare degli anni, la scelta del TAC è sempre più frequente.

Già in una metanalisi del $2005^{10}$ il TAC riduceva significativamente il rischio di rigetto acuto rispetto alla ciclosporina. Questi dati sono poi stati confermati dallo studio Symphony. ${ }^{11}$

Entrambi i farmaci comportano tuttavia importanti effetti collaterali.

Il TAC, rispetto alla ciclosporina, ha maggiori effetti collaterali neurologici, maggior incidenza di diabete mellito, comporta più frequentemente alopecia, mentre la ciclosporina ha più frequentemente irsutismo, iperplasia gengivale e ipertensione.

Entrambi i farmaci interagiscono con molti medicamenti. Un aumento dei livelli di TAC o CyA è stato osservato con la contemporanea somministrazione dei bloccanti del calcio nicardipinici, con agenti antifungini, con alcuni antibiotici come l'eritromicina. Al contrario anticonvulsivanti o alcuni farmaciantitubercolaririduconoilivellideglianticalcineurinici.

\section{Scelta di un agente antimetabolico}

Di fatto MMF e EC-MPS hanno quasi completamente soppiantato l'azatioprina dopo che una gran quantità di studi ha documentato una loro maggiore efficacia. In aggiunta, l'azatioprina comporta più frequentemente gravi effetti collaterali come la leucopenia.

\section{Riduzione o sospensione dello steroide}

Tutti i centri trapianto riducono col tempo la somministrazione dello steroide fino ad arrivare a $5 \mathrm{mg} / \mathrm{die}$.

È molto discussa la sospensione completa dello steroide. Questa da un lato ridurrebbe gli effetti collaterali dovuti agli steroidi, dall'altro potrebbe favorire l'insorgenza di rigetti acuti tardivi. Probabilmente la sospensione dello steroide, a parte i trials randomizzati, controllati, dovrebbe essere decisa caso per caso secondo il rischio immunologico deipazienti,dellafunzionerenale, dell'immunosoppressione globale, della gravità di effetti collaterali dovuti agli steroidi.

L'uso ab initio degli inibitori dell'mTOR al fine di ridurre il dosaggio degli inibitori della calcineurina è poco comune negli Stati Uniti, mentre alcuni studi europei hanno fornito ottimi risultati. ${ }^{12}$ Una revisione della Cochrane ${ }^{13}$ non ha permesso di concludere a questo riguardo.

\section{Belatacept}

Molti studi hanno valutato l'efficacia e la sicurezza del Belatacept. Il farmaco, bloccando il secondo segnale della co-stimolazione, permette di non somministrare gli inibitori della calcineurina, evitando così tutti gli effetti collaterali collegati a questi ultimi.

Nello studio BENEFIT ${ }^{14}$ i pazienti trattati con Belatacept avevano più elevata incidenza di rigetti a 12 mesi rispetto alla ciclosporina, ma una migliore funzione renale persistente anche dopo 7 anni dal trapianto.

Questi dati sono stati confermati dallo studio BENEFIT EXT praticato su pazienti trapiantati con reni provenienti da donatori marginali. ${ }^{15}$

$\mathrm{Si}$ aveva nuovamente maggiore incidenza di rigetti acuti, ma migliore funzione renale persistente nel tempo.

Un problema comune ad entrambi gli studi è stata una maggiore incidenza di malattie linfoproliferative (Posttransplant lymphoproliferative disease, PTLD) nei pazienti trattati con Belatacept, in particolare nei pazienti negativi per virus di Epstein-Barr.

\section{Conclusioni}

In questa rassegna sono stati trattati solo i farmaci in commercio e più frequentemente usati. Altri farmaci sono impiegati in caso di complicanze in corso di trapianto e saranno trattati in rassegne successive.

Da tempo siamo in attesa di nuovi farmaci, in particolare di farmaci biologici. Quello che rallenta lo sviluppo di nuovi farmaci nel trapianto è l'orientamento delle diverse aziende farmaceutiche più orientato allo sviluppo di farmaci per patologie più diffuse. È da attendersi, come è accaduto per il Rituximab, che nuovi farmaci per il trapianto possano essere attinti da farmaci approvati per altre patologie come neoplasie, malattie reumatiche, etc.

\section{Dichiarazione di assenza di conflitto di interessi}

Gli Autori dichiarano di non avere conflitti di interessi.

\section{Finanziamenti}

Gli Autori dichiarano di non aver ricevuto finanziamenti specifici da qualsiasi ente nei settori pubblico, privato o senza fini di lucro.

\section{Bibliografia}

1. Cai $\mathrm{J}$ and Terasaki PI. Induction immunosuppression improves long-term graft and patient outcome in organ transplantation: an analysis of United Network for Organ Sharing registry data. Transplantation 2010;90(12):15111515. 
2. Morris PJ and Russell NK. Alemtuzumab (Campath-1H): a systematic review in organ transplantation. Transplantation. 2006;81(10):1361-1367.

3. van den Hoogen MW, Kamburova EG, Baas MC, et al. Rituximab as induction therapy after renal transplantation: a randomized, double-blind, placebo-controlled study of efficacy and safety. Am J Transplant 2015;15(2): 407-416.

4. Vincenti F, Kirkman R, Light S, et al. Interleukin-2-receptor blockade with daclizumab to prevent acute rejection in renal transplantation. Daclizumab Triple Therapy Study Group. $N$ Engl J Med 1998;338(3):161-165.

5. Mourad G, Garrigue V, Squifflet JP, et al. Induction versus noninduction in renal transplant recipients with tacrolimusbased immunosuppression. Transplantation 2001;72(6): 1050-1055.

6. Brennan DC, Daller JA, Lake KD, et al. Rabbit antithymocyte globulin versus basiliximab in renal transplantation. $N$ Engl J Med. 2006;355(19):1967-1977.

7. Wong W, Agrawal N, Pascual M, et al. Comparison of two dosages of thymoglobulin used as a short-course for induction in kidney transplantation. Transpl Int. 2006;19(8):629-635.

8. Kidney Disease: Improving Global Outcomes (KDIGO) Transplant Work Group. KDIGO clinical practice guidline for the care of kidney transplant recipients. Am J Transplant 2009;9 (Suppl 3:S1).
9. Webster AC, Ruster LP, McGee R, et al. Interleukin 2 receptor antagonists for kidney transplant recipients. Cochrane Database Syst Rev 2010;(1):CD003897.

10. Webster AC, Woodroffe RC, Taylor RS, et al. Tacrolimus versus ciclosporin as primary immunosuppression for kidney transplant recipients: meta-analysis and meta-regression of randomised trial data. $B M J$ 2005;331(7520):810.

11. Ekberg H, Tedesco-Silva H, Demirbas A, et al. Reduced exposure to calcineurin inhibitors in renal transplantation. $N$ Engl J Med 2007;357(25):2562-2575.

12. Pascual J, Berger SP, Witzke O, et al. Everolimus with Reduced Calcineurin Inhibitor Exposure in Renal Transplantation. J Am Soc Nephrol 2018;29(7):19791991.

13. Webster AC, Lee VW, Chapman JR, et al. Target of rapamycin inhibitors (sirolimus and everolimus) for primary immunosuppression of kidney transplant recipients: a systematic review and meta-analysis of randomized trials. Transplantation 2006;81(9):1234-1248.

14. Vincenti F, Charpentier B, Vanrenterghem Y, et al. A phase III study of belatacept-based immunosuppression regimens versus cyclosporine in renal transplant recipients (BENEFIT study). Am J Transplant 2010;10(3):535-546.

15. Durrbach A, Pestana JM, Pearson T, et al. A phase III study of belatacept versus cyclosporine in kidney transplants from extended criteria donors (BENEFIT-EXT study). Am J Transplant 2010;10(3):547-557. 